\title{
DIABETES
}

\section{Roux-en-Y rescues islet function via PYY}

The beneficial effects of Roux-en-Y gastric bypass (RYGB) surgery in patients with type 2 diabetes mellitus (T2DM) might be mediated by the gut hormone peptide YY (PYY), according to new research.

"Gastric bypass surgery results in rapid remission of T2DM in $>80 \%$ of patients, without the need for any diabetes medication," explains lead author Reshma Ramracheya, "but the impact of bypass surgery on islet function has not been explored in depth."

The team performed RYGB surgery in diabetic Goto-Kakizaki rats, which improved glucose-stimulated insulin secretion and glucagon suppression compared with sham-operated rats, and also reversed the morphological changes associated with T2DM in rat pancreatic islets. When the researchers cultured diabetic rat islets with sera obtained from RYGB-treated animals, both insulin secretion and glucagon suppression were also improved, suggesting that a blood-borne factor mediates the effects of RYGB surgery.

Ramracheya and colleagues first suspected the involvement of glucagon-like peptide 1 (GLP-1), which has been implicated in RYGB-mediated T2DM remission; but no difference in GLP-1 levels was found in sera from RYGB-treated rats and improved islet function persisted in the presence of a GLP-1 receptor antagonist. By contrast, PYY levels were markedly increased in serum from RYGB-treated diabetic rats. Importantly, PYY treatment restored islet cell function in diabetic rats and enhanced glucose-stimulated insulin secretion and glucagon suppression in islets isolated from healthy humans.

"The next step is to explore the translational potential of these findings," concludes Ramracheya, "Gastric bypass procedures remain risky, expensive and unsuitable for many patients... a pharmacological agent enhancing PYY release or its action could provide an effective and non-surgical therapy for T2DM."

Charlotte Ridler

ORIGINAL ARTICLE Ramracheya, R. D. et al.PYY

dependent restoration of impaired insulin and

glucagon secretion in type 2 diabetes following

Roux-en-Y gastric bypass surgery. Cell Rep. http:// dx.doi.org/10.1016/j.celrep.2016.03.091 (2016) 